\title{
Mobile robots communication and control framework for USARSim
}

\begin{abstract}
In recent years there have been intensive efforts in robotics researches from the earliest stages of education. Subject of this paper is a powerful mobile robot communication and control framework for USARSim simulator that can be used both for research and education. Mobile Robots Communication and Control Framework (MCCF) is developed in order to offer faster and easier communication process with the USARSim server within Matlab that differentiates it from most existing basic open source control interfaces. Most notably, it takes the advantages of easy integration with other analysis and control methods that have been provided in Matlab tool-boxes. MCCF enables communication and control of a wide range of robots platforms including but not limited to wheeled-robots, legged-robots, submarine robots and aerial robots. In this paper we describe its general architecture, features and examples of utilization for researchers who are interested in mobile robot simulations for education and research.
\end{abstract}

